\title{
Impact of caveolin- I expression on prognosis of pancreatic ductal adenocarcinoma
}

\author{
M Suzuoki*,', M Miyamoto', K Kato', K Hiraoka', T Oshikiri', Y Nakakubo', A Fukunaga', T Shichinohe', \\ T Shinohara', T Itoh', S Kondo' and H Katoh'
}

'Department of Surgical Oncology, Division of Cancer Medicine, Hokkaido University Graduate School of Medicine, Kita- 15, Nishi-7, Kita-ku, Sapporo, Hokkaido 060-8638 Japan; ${ }^{2}$ Department of Pathology, Teinekeijinkai Hospital, I-I 2, Maeda, Teine-ku, Sapporo, 006-08/I Japan; ${ }^{3}$ Department of Surgical Pathology, Hokkaido University Hospital, Kita-14, Nishi-5, Kita-ku, Sapporo, 060-8648 Japan

\begin{abstract}
Caveolin-I is a major component of caveolae and plays a regulatory role in several signalling pathways. Caveolin-I was recently identified as a metastasis-related gene in prostate cancer. The clinical effects of caveolin-I expression in pancreatic carcinoma, however, remain unknown. In this study, we have investigated the relationship between caveolin-I expression and the clinicopathologic variables and clinical outcome in 79 patients with pancreatic adenocarcinoma undergoing surgical resection. Caveolin-I expression was determined by immunohistochemistry, using a polyclonal anti-caveolin-I antibody. Patients were divided into two groups based on the extent of caveolin-I expression: a negative expression group (immunoreactivity in less than 50\% of cells) and a positive expression group. Positive caveolin-I immunostaining was detected in 32 cases (40.5\% of total), while non-neoplastic ductal epithelium showed little or no staining. Positive caveolin-I expression was correlated with tumour diameter $(P=0.0079)$, histopathologic grade $(P=0.0272)$ and poor prognosis $(P=0.0008)$. Upon multivariate analysis with Cox's proportional hazards model, positive caveolin- I expression was shown to be an independent negative predictor for survival $(P=0.0358)$. These results suggest that caveolin-I overexpression is associated with tumour progression, thereby indicating a poor prognosis for certain patients undergoing surgical resection for pancreatic carcinoma. British Journal of Cancer (2002) 87, || 40 - | |44. doi:| 0.1038/sj.bjc.66006I9 www.bjcancer.com
\end{abstract}

(c) 2002 Cancer Research UK

Keywords: caveolin-I; pancreatic carcinoma; immunohistochemistry; prognosis

Pancreatic carcinoma is one of the most common and lethal malignancies (Niederhuber et al, 1995). Despite developments in surgical and non-surgical therapies, significant improvements in long-term survival have not been realized. The majority of patients present an advanced disease at the time of diagnosis, because of the tumour's characteristic rapid progression and late detectability. Accordingly, the resectability rate for pancreatic carcinoma is low in most series. Tumour resection is performed in only $9-36 \%$ of all patients. Even in those patients fortunate enough to have a resectable lesion, the 5-year survival rate following resection is $11-24 \%$ (Conlon et al, 1996; Yamamoto et al, 1998; Sener et al, 1999). The reasons behind the aggressiveness of pancreatic carcinoma are not clearly understood. Therefore, the identification of biological markers that correlate with clinicopathologic variables or prognosis is important in understanding the characteristics of this neoplasm and selecting patients who would benefit most from multimodality treatment.

The $21-24 \mathrm{kDa}$ molecule, caveolin-1, is a major structural component of caveolae, $50-100 \mathrm{~nm}$ vesicular invaginations of the plasma membrane that mediate molecular transport and signal transduction activities. Caveolin-1 plays a regulatory role in several signalling pathways, including the Src family tyrosine kinase, epidermal growth factor receptor, Neu/HER2 (c-erbB2), protein kinase $\mathrm{C}$, transforming growth factor (TGF)- $\beta / \mathrm{SMAD}$, and Wnt/

*Correspondence: Dr M Suzuoki; E-mail: suzuoki@med.hokudai.ac.jp Received 4 April 2002; revised 22 August 2002; accepted 9 September 2002 beta-catenin/lef-1 pathways (Engelman et al, 1998a; Galbiati et al, 2000; Razani et al, 2001). In prostate cancer, caveolin-1 has been found to be a metastasis-related gene with an independent prognostic value for patients following radical prostatectomy (Yang et al, 1999). Recent study has also found an association between caveolin-1 expression and less favourable prognoses in oesophageal squamous cell carcinoma (Kato et al, 2002). On the other hand, caveolin-1 has been reported as a tumour suppressor gene (Engelman et al, 1998b) and its expression level is reduced in several cancers (Lee et al, 1998; Racine et al, 1999; Bender et al, 2000; Wiechen et al, 2001a,b). Thus, the role of caveolin-1 in tumour remains controversial.

In the present study, we examined the expression and clinical impact of caveolin-1 in a cohort of patients with pancreatic carcinoma, using immunohistochemical analysis. We found that caveolin-1 is overexpressed in pancreatic carcinoma and has a significant prognostic value for patients with this disease.

\section{MATERIALS AND METHODS}

\section{Patients and specimens}

We studied surgically-resected specimens from 79 patients with pancreatic ductal adenocarcinomas and seven with chronic pancreatitis treated at the Department of Surgical Oncology of Hokkaido University Hospital, the Department of Surgery of Teinekeijinkai Hospital, and the Department of Surgery of Hokkaido Gastroenterology Hospital from 1992 to 1999. Among the 79 
patients with cancer, 60 underwent pancreatico-duodenectomy (Whipple operation), 14 underwent distal pancreatecomy and five underwent total pancreatectomy with curative intent. All patients received extended radical lymphadenectomy. Pancreatic resection was not performed in patients with distant site metastases. Any cases of cystadenocarcinoma or mucin-producing carcinoma were excluded from this study. Pancreatic carcinoma tissues were obtained from 44 men and 35 women with a median age of 63 years (range, 31-83 years). The median duration of follow-up was 57.6 months (range, 3.8-103.8 months), and 59 patients $(74.7 \%)$ died during the follow-up period.

All specimens were fixed in $10 \%$ formalin and embedded in paraffin wax. Representative blocks were selected (based primarily on the greatest dimensions of the tumour), and serial $4 \mu \mathrm{m}$-thick sections were examined by immunohistochemistry. Histological classification of tumours was based on the World Health Organization criteria. All tumours were staged according to the pTNM pathological classification of the UICC (International Union Against Cancer) (Sobin and Wittekind, 1997). Thirty of 79 patients had positive resection margin for carcinoma, as evaluated microscopically.

\section{Immunohistochemistry}

Immunohistochemical reactions were carried out using the streptavidin-biotin-peroxidase method. Sections were deparaffinized in xylene and rehydrated through a graded series of ethanol/water. Endogenous peroxidase was blocked with 3\% hydrogen peroxide for $10 \mathrm{~min}$. Sections were washed twice in phosphate-buffered saline (PBS) and incubated with 10\% normal goat serum (Histofine SAB-PO kit; Nichirei, Tokyo, Japan) for $30 \mathrm{~min}$. Primary antibody (anti-caveolin-1 rabbit polyclonal antibody directed against caveolin-1 residues 2-21, Santa Cruz Biotechnology, Santa Cruz, CA, USA) was applied in a 1:400 dilution in PBS, and sections were incubated overnight at $4^{\circ} \mathrm{C}$. After three additional washes, sections were incubated with polyvalent biotinylated goat anti-rabbit antibody for $30 \mathrm{~min}$ at room temperature. Sections were washed three times in PBS and incubated with streptavidinconjugated peroxidase for $30 \mathrm{~min}$ at room temperature. After three additional washes, the reaction product was visualized after incubating with 3,3'-diaminobenzidine tetrahydrochloride (Histofine SAB-PO kit; Nichirei, Tokyo, Japan) for approximately $15 \mathrm{~min}$ and then washing with distilled water. Sections were counterstained in haematoxylin for $1 \mathrm{~min}$ and mounted in Permount (Micro Slides; Muto-Glass, Tokyo, Japan). The smooth muscle cell segments or endothelium, both known to be abundant in caveolin-1, were used as positive controls. For a negative control, nonimmune purified rabbit serum was used for the primary antibody. The number of stained cells per 1000 was determined under a microscope (Olympus Optical Co, Ltd, Tokyo, Japan) in three visual fields, at a magnification of $\times 200$. When the total number of cancer cells observed under microscope was less than 1000 , all cells were counted. When over $50 \%$ of all cancer cell cytoplasm was stained, the tumour was considered caveolin-1 positive. This cut-off value $(50 \%)$ was adopted from previous reports (Yang et al, 1999; Kato et al, 2002). The present study was performed retrospectively, but all specimens were evaluated by three investigators who were blind to the patients' clinical information.
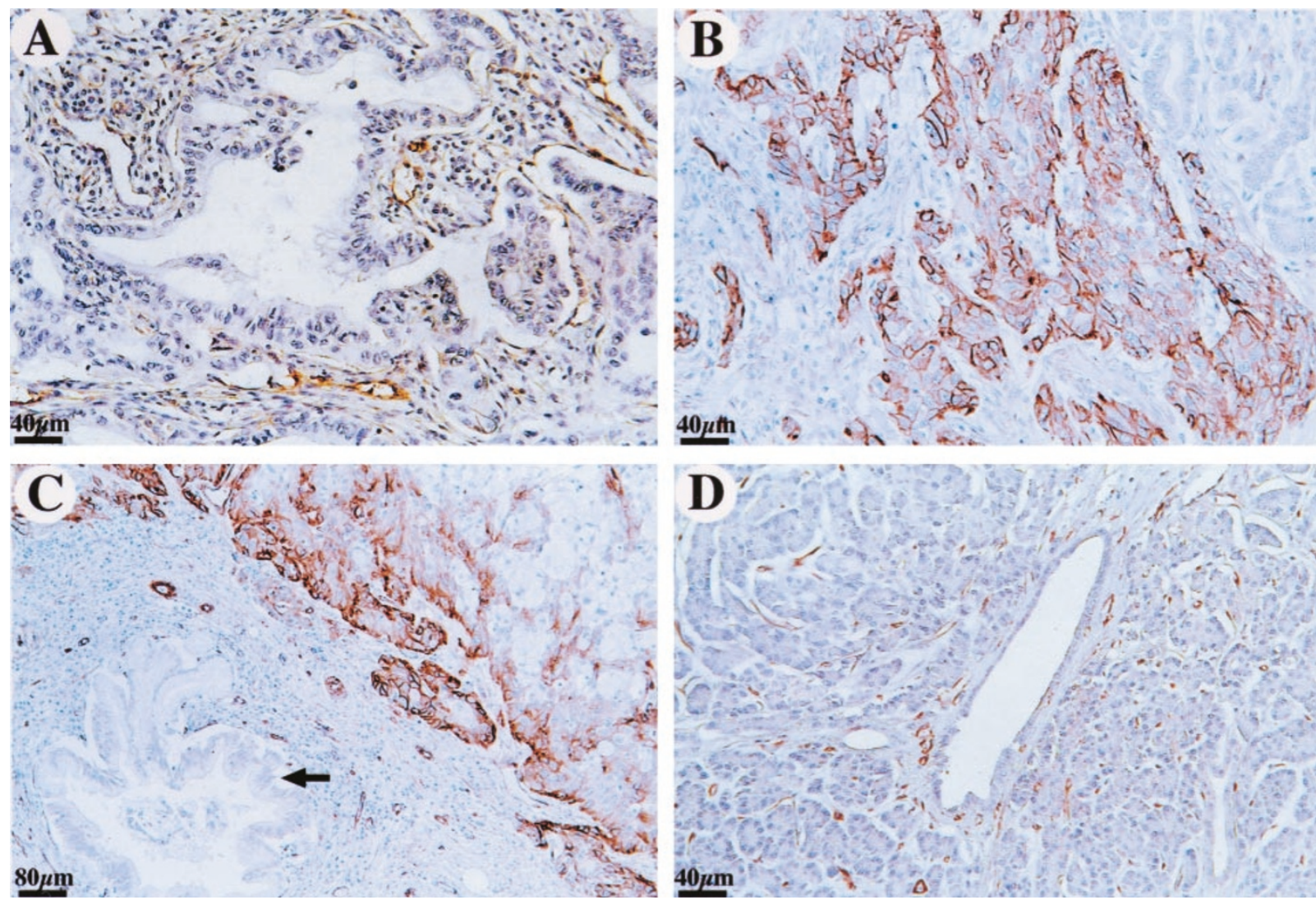

Figure I Representative examples of caveolin-I immunostaining. (A) Caveolin-I immunoreactivity was observed in less than $5 \%$ of cancer cells, while endothelial cells showed strong staining as an internal control. (B) Strong staining both in the cytoplasm and on the membrane of cancer cells. Over $50 \%$ of tumour cells were reactive. (C) Non-neoplastic ductal epithelium adjacent to cancer cells showed no immunoreactivity (arrow). (D) In chronic pancreatitis, normal ductal epithelium showed no immunoreactivity. (Original magnifications: A, B, D, $\times 200 ; \mathbf{C}, \times 100$ ) 


\section{Statistical analysis}

Caveolin-1 immunoreactivity was assessed for association with clinicopathologic variables using the following statistical tests: the Mann - Whitney test for depth of invasion, pTNM stage and histopathologic grade; the chi-square test or Fisher's exact test for the remaining variables. The Kaplan-Meier method was used to generate survival curves, and survival differences were analysed with the log-rank test, based on the status of caveolin-1 expression. Univariate and multivariate analyses were performed using Cox's proportional hazard regression model. Probability values less than 0.05 were considered statistically significant. All analyses were performed using statistical analysis software (StatView, version 5.0; SAS Institute, Inc. Cary, NC, USA).

\section{RESULTS}

\section{Caveolin-1 expression of pancreatic carcinoma and chronic pancreatitis specimens}

Caveolin-1 was expressed both on the cell membrane and in the cytoplasm of cancer cells, as evidenced by the presence of stained granular immunoreaction products. Out of the 79 specimens of pancreatic carcinoma, seventeen specimens were immunoreactive for caveolin-1 in less than 5\% of cells (Figure 1A); 20 specimens were immunoreactive in $5-25 \%$ of cells; 10 specimens were immunoreactive in 25-50\% of cells; and 32 specimens were immunoreactive in over $50 \%$ of cells (Figure 1B). According to our criteria, 32 out of $79(40.5 \%)$ tumours were positive for caveolin-1. Histologically non-neoplastic ductal epithelium adjacent to cancer cells showed little or no immunoreactivity with caveolin-1 (Figure 1C). In all chronic pancreatitis specimens, normal ductal epithelium showed little or no staining, while the smooth muscle and endothelial cells consistently stained positively (Figure 1D).

\section{Caveolin-1 expression and clinicopathologic variables (Table 1)}

Caveolin-1 expression showed a significant correlation with tumour diameter $(P=0.0079)$ and histopathologic grade $(P=0.0272)$. No significant association was noted between caveolin-1 expression and other clinicopathologic variables.

\section{Caveolin-1 expression and prognosis}

Survival curves of patients, grouped according to level of caveolin1 staining, are shown in Figure 2. The caveolin-1 positive group had a significantly poorer prognosis than the caveolin-1 negative group (3-year survival rate was $4.8 \%$ vs $33.8 \%$, respectively) (logrank test: $P=0.0008)$. Upon univariate analysis with Cox's proportional hazards model, lymph node metastasis $(P=0.0007)$, tumour diameter $(P=0.0027)$, positive surgical margin $(P=0.0014)$ and caveolin-1 immunopositivity $(P=0.0011)$ were all positively correlated with poor prognosis. Multivariate analyses indicated that caveolin-1 positivity was an independent unfavourable prognostic factor $(P=0.0358$; risk ratio, $1.880 ; 95 \% \mathrm{CI}, 1.043-3.390)$, as were the presence of lymph node metastases $(P=0.0009)$ and a positive surgical margin $(P=0.0045)$ (Table 2$)$.

\section{DISCUSSION}

The present study shows that: (1) the level of caveolin-1 expression is elevated in pancreatic adenocarcinoma relative to non-neoplastic ductal epithelium, (2) caveolin-1 expression is related to tumour diameter and histopathologic grade, and (3) positive caveolin-1 expression is an independent unfavourable prognostic factor following surgical resection.
Table I Association between caveolin-I expression and clinicopathological variables

\begin{tabular}{|c|c|c|c|c|}
\hline \multirow[b]{2}{*}{ Variables } & \multirow[b]{2}{*}{ No. of cases } & \multicolumn{2}{|c|}{ Caveolin-I } & \multirow[b]{2}{*}{$P$ value } \\
\hline & & $\begin{array}{c}\text { Negative (\%) } \\
n=47\end{array}$ & $\begin{array}{c}\text { Positive (\%) } \\
\text { n=32 }\end{array}$ & \\
\hline Age (years) & & & & 0.8476 \\
\hline$<63$ & 36 & $21(58)$ & $15(42)$ & \\
\hline$\geqslant 63$ & 43 & $26(60)$ & $17(40)$ & \\
\hline Gender & & & & 0.7042 \\
\hline Male & 44 & $27(6 \mid)$ & $17(39)$ & \\
\hline Female & 35 & $20(57)$ & $15(43)$ & \\
\hline $\mathrm{p} T$ & & & & 0.0557 \\
\hline 2 & 4 & $3(75)$ & I (25) & \\
\hline 3 & 50 & $33(66)$ & $17(34)$ & \\
\hline 4 & 25 & II (44) & $14(56)$ & \\
\hline $\mathrm{pN}$ & & & & 0.2425 \\
\hline 0 & 23 & $16(70)$ & $7(30)$ & \\
\hline I & 56 & $31(55)$ & $25(45)$ & \\
\hline Stage (pTNM) & & & & 0.0649 \\
\hline$\|$ & 17 & $12(7 \mid)$ & $5(29)$ & \\
\hline III & 37 & $24(65)$ & $13(35)$ & \\
\hline IVA & 25 & $11(44)$ & $14(56)$ & \\
\hline Histopathologic Grade & & & & $0.0272 *$ \\
\hline 1 & 25 & $19(76)$ & $6(24)$ & \\
\hline 2 & 46 & $25(54)$ & $21(45)$ & \\
\hline 3 & 8 & $3(37)$ & $5(63)$ & \\
\hline Lymphatic invasion & & & & 0.4340 \\
\hline Negative & 13 & $9(69)$ & $4(3 \mid)$ & \\
\hline Positive & 66 & $38(58)$ & $28(42)$ & \\
\hline Venous invasion & & & & 0.0644 \\
\hline Negative & 32 & $23(72)$ & $9(28)$ & \\
\hline Positive & 47 & $24(5 \mathrm{I})$ & $23(49)$ & \\
\hline Tumour diameter & & & & $0.0079 *$ \\
\hline$\leqslant 3.0 \mathrm{~cm}$ & 39 & $29(74)$ & $10(26)$ & \\
\hline$>3.0 \mathrm{~cm}$ & 40 & $18(45)$ & $22(55)$ & \\
\hline Surgical margin & & & & 0.3828 \\
\hline Negative & 49 & $31(63)$ & $18(37)$ & \\
\hline Positive & 30 & $16(53)$ & $14(47)$ & \\
\hline
\end{tabular}

*Significant; pT, depth of invasion (pTNM); pN, lymph node metastasis (pTNM).

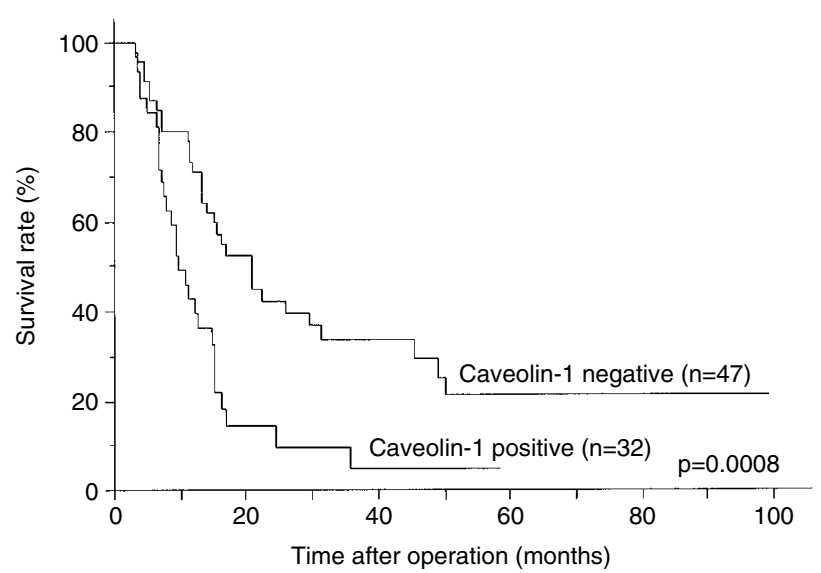

Figure 2 Kaplan-Meier analysis of the overall survival of patients with negative and positive tumour caveolin-I expression (log-rank test, $P=0.0008)$.

The expression of caveolin-1 is elevated in various cancer tissues, including prostate cancer, oesophageal squamous cell carcinoma, colon cancer and breast cancer (Yang et al, 1998, 1999; Fine et 
Table 2 Prognostic factors in Cox's proportional hazards model

\begin{tabular}{|c|c|c|c|c|c|c|}
\hline \multirow[b]{2}{*}{ Variables } & \multicolumn{3}{|c|}{ Univariate } & \multicolumn{3}{|c|}{ Multivariate } \\
\hline & Risk ratio & $95 \% \mathrm{Cl}$ & $P$ value & Risk ratio & $95 \% \mathrm{Cl}$ & $P$ value \\
\hline \multicolumn{7}{|l|}{ Age (years) } \\
\hline$\leqslant 1>63$ & 1.328 & $0.796-2.214$ & 0.2767 & & & \\
\hline \multicolumn{7}{|l|}{ Gender } \\
\hline Male/Female & 1.189 & $0.708-1.997$ & 0.5128 & & & \\
\hline \multicolumn{7}{|l|}{ pT } \\
\hline $2 / 3-4$ & 1.318 & $0.408-4.255$ & 0.6443 & & & \\
\hline \multicolumn{7}{|l|}{$\mathrm{pN}$} \\
\hline $1 / 0$ & 3.150 & $1.625-6.106$ & $0.0007 *$ & 3.252 & $1.618-6.536$ & $0.0009 *$ \\
\hline \multicolumn{7}{|c|}{ Histopathologic grade } \\
\hline $2-3 / 1$ & 1.584 & $0.899-2.789$ & 0.1113 & & & \\
\hline \multicolumn{7}{|c|}{ Lymphatic invasion } \\
\hline$+1-$ & 1.800 & $0.852-3.802$ & 0.1235 & & & \\
\hline \multicolumn{7}{|l|}{ Venous invasion } \\
\hline$+1-$ & 1.665 & $0.979-2.831$ & 0.0599 & & & \\
\hline \multicolumn{7}{|c|}{ Tumour diameter $(\mathrm{cm})$} \\
\hline$>1 \leqslant 3.0$ & 2.247 & $1.325-3.812$ & $0.0027 *$ & 1.397 & $0.790-2.472$ & 0.2506 \\
\hline \multicolumn{7}{|l|}{ Surgical margin } \\
\hline$+1-$ & 2.394 & $|.40|-4.09 \mid$ & $0.0014 *$ & 2.260 & $1.287-3.970$ & $0.0045 *$ \\
\hline \multicolumn{7}{|l|}{ Caveolin-I } \\
\hline$+1-$ & 2.415 & $1.422-4.099$ & $0.0011 *$ & 1.880 & $1.043-3.390$ & $0.0358 *$ \\
\hline
\end{tabular}

*Significant; Cl, confidence interval.

al, 2001; Kato et al, 2002). In addition, a correlation between caveolin-1 expression and clinicopathological variables has been shown in these cases. In prostate cancer, caveolin-1 expression positively correlated with Gleason score, positive surgical margin and lymph node metastasis (Yang et al, 1999). In oesophageal squamous cell carcinoma, expression of caveolin-1 was positively correlated with histopathologic stage, lymph node metastasis and distant metastasis (Kato et al, 2002). In the present study, positive caveolin-1 expression was detected in 32 out of 79 tumours $(40.5 \%)$ in pancreatic carcinoma, while non-neoplastic ductal epithelium showed little or no immunoreactivity. Moreover, caveolin-1 immunopositivity is positively correlated with tumour diameter and histopathologic grade in this cancer as well. These results suggest that caveolin-1 overexpression may contribute to the aggressiveness of pancreatic carcinoma.

Except for the case of prostate cancer, however, the molecular and cellular underpinnings of the relationship between caveolin-1 expression and cancer progression remain unclear. In prostate cancer, caveolin-1 both protects against androgen withdrawalinduced apoptosis in vitro and in vivo (Nasu et al, 1998) and blocks c-myc-induced apoptosis in cancer cells (Timme et al, 2000). Moreover, caveolin-1 mediates testosterone-stimulated survival/ clonal growth and promotes metastatic activity (Li et al, 2001), while overexpression of caveolin-1 potentiates ligand-dependent androgen receptor activation (Lu et al, 2001). It is well known that prostate cancer is androgen-dependent. Androgen receptor has also been detected in cancerous tissues of pancreatic carcinoma (Corbishley et al, 1986), and it is thought that testosterone increases growth of this tumour (Greenway, 1998). Thus, a similar mechanism may be at work in prostate cancer and pancreatic carcinoma.

Interestingly, it has recently been reported that a caveolin-1 mutation at codon 132 was found in human breast cancer specimens and that the mutated caveolin-1 induced cellular transformation, activated the mitogen-activated protein kinase (MAPK)-signalling pathway, and promoted invasion ability in NIH3T3 cells (Hayashi et al, 2001). Following up this result, we searched for this caveolin-1 mutation in 11 pancreatic cancer cell lines, but found no mutation in the predicted functional domains (including the scaffolding and membrane-spanning domain) (data not shown). In light of this finding, we suggest that overexpression of wild-type caveolin-1 may be associated with cancer progression in pancreatic carcinoma.

Contrary to the above-mentioned studies, it has been reported that caveolin-1 levels were reduced in a variety of cancer cell lines and cancer specimens (including human breast cancer, lung cancer, colon cancer, ovarian cancer and sarcoma) (Lee et al, 1998; Racine et al, 1999; Bender et al, 2000; Wiechen et al, 2001a,b). Under some conditions, caveolin-1 has been shown to suppress growth of specific cell lines in vitro and in vivo (Koleske et al, 1995; Engelman et al, 1997; Suzuki et al, 1998), and some have suggested that caveolin-1 functions as a tumour suppressor gene (Engelman et al, 1998b). The reasons behind this seemingly contradictory evidence remain unclear. Lee et al (2000) suggest that the diverse effects of caveolin-1 may be mediated by different regions of the caveolin-1 molecule, and may depend on the expression levels of other coexpressed molecules. It has been reported that the oncosuppressive effect of caveolin-1 is mediated through the caveolin-1 scaffolding domain (residues 82-101) (Okamoto et al, 1998). c-Src, however, induces phosphorylation of caveolin-1 at residue tyrosine 14. Tyrosine 14-phosphorylated caveolin-1 confers binding to growth factor receptor-binding protein 7 (Grb7) and augments both anchorage-independent growth and epidermal growth factor (EGF)-stimulated cell migration (Lee et al, 2000). In pancreatic carcinoma, Src kinase overexpression and activation has been reported (Lutz et al, 1998). Thus, caveolin-1 might cooperate with other molecules, such as c-Src and Grb7, to stimulate tumour growth in pancreatic carcinoma.

This is the first study demonstrating the prognostic significance of caveolin-1 expression in pancreatic carcinoma. The 3-year survival rate following surgical resection of the caveolin-1 negative group was $33.8 \%$, while that in the caveolin-1 positive group was only $4.8 \%$. Furthermore, multivariate analysis demonstrated that positive caveolin-1 expression is an independent negative prognostic factor. These results can increase the accuracy of prognosis for patients with pancreatic carcinoma, following surgical resection. Caveolin-1 overexpression in resected specimens may be a useful index of adjuvant therapy for the patients with a high risk of poor prognosis. 
In summary, overexpression of caveolin-1 in pancreatic carcinoma may contribute to tumour progression and be a negative prognostic predictor following surgery. For patients with a tumour overexpressing caveolin-1, closer follow-up should be performed to find recurrence, and adjuvant therapy may be beneficial. However, at present, the role of caveolin-1 in pancreatic carcinoma remains unclear, and elucidation awaits further investigation.

\section{REFERENCES}

Bender FC, Reymond MA, Bron C, Quest AF (2000) Caveolin-1 levels are down-regulated in human colon tumors, and ectopic expression of caveolin-1 in colon carcinoma cell lines reduces cell tumorigenicity. Cancer Res 60: $5870-5878$

Conlon KC, Klimstra DS, Brennan MF (1996) Long-term survival after curative resection for pancreatic ductal adenocarcinoma. Clinicopathologic analysis of 5-year survivors. Ann Surg 223: 273-279

Corbishley TP, Iqbal MJ, Wilkinson ML, Williams R (1986) Androgen receptor in human normal and malignant pancreatic tissue and cell lines. Cancer 57: $1992-1995$

Engelman JA, Wykoff CC, Yasuhara S, Song KS, Okamoto T, Lisanti MP (1997) Recombinant expression of caveolin-1 in oncogenically transformed cells abrogates anchorage-dependent growth. J Biol Chem 272: $16374-16381$

Engelman JA, Lee RJ, Karnezis A, Bearss DJ, Webster M, Siegel P, Muller WJ, Windle JJ, Pestell RG, Lisanti MP (1998a) Reciprocal regulation of Neu tyrosine kinase activity and caveolin-1 protein expression in vitro and in vivo. Implications for human breast cancer. J Biol Chem 273: 20448 - 20455

Engelman JA, Zhang XL, Galbiati F, Lisanti MP (1998b) Chromosomal localization, genomic organization, and developmental expression of murine caveolin gene family (Cav-1, -2 , and -3): Cav-1 and Cav-2 genes map to a known tumor suppressor locus (6-A2/7q31). FEBS Lett 429: $330-336$

Fine SW, Lisanti MP, Galbiati F, Li M (2001) Elevated expression of caveolin1 in adenocarcinoma of the colon. Am J Clin Pathol 115: 719-724

Galbiati F, Volonte D, Brown AMC, Weinstein DE, Ben-Ze'ev A, Pestell RG, Lisanti MP (2000) Caveolin-1 expression inhibits Wnt/ $\beta$-Catenin/Lef-1 signaling by recruiting $\beta$-Catenin to Caveolae membrane domains. J Biol Chem 275: $23368-23377$

Greenway BA (1998) Effect of flutamide on survival in patients with pancreatic cancer: results of a prospective, randomised, double blind, placebo controlled trial. BMJ 316: $1935-1938$

Hayashi K, Matsuda S, Machida K, Yamamoto T, Fukuda Y, Nimura Y, Hayakawa T, Hamaguchi M (2001) Invasion activating caveolin-1 mutation in human scirrhous breast cancers. Cancer Res 61: 2361-2364

Kato K, Hida Y, Miyamoto M, Hashida H, Shinohara T, Itoh T, Okushiba S, Kondo S, Katoh H (2002) Overexpression of caveolin-1 in esophageal squamous cell carcinoma correlates with lymph node metastasis and pathologic stage. Cancer 94: 929-933

Koleske AJ, Baltimore D, Lisanti MP (1995) Reduction of caveolin and caveolae in oncogenically transformed cells. Proc Natl Acad Sci USA 92: 1381 1385

Lee H, Volonte D, Galbiati F, Iyengar P, Lublin DM, Bregman DB, Wilson MT, Campos-Gonzalez R, Bouzahzah B, Pestell RG, Scherer PE, Lisanti MP (2000) Constitutive and growth factor-regulated phosphorylation of caveolin-1 occurs at the same site (Tyr-14) in vivo: identification of a cSrc/Cav-1/Grb7 signaling cassette. Mol Endocrinol 14: 1750-1775

Lee SW, Reimer CL, Oh P, Campbell DB, Schnitzer JE (1998) Tumor cell growth inhibition by caveolin re-expression in human breast cancer cells. Oncogene 16: $1391-1397$

Li L, Yang G, Ebara S, Satoh T, Nasu Y, Timme TL, Ren C, Wang J, Tahir SA, Thompson TC (2001) Caveolin-1 mediates testosterone-stimulated survival/clonal growth and promotes metastatic activities in prostate cancer cells. Cancer Res 61: $4386-4392$

\section{ACKNOWLEDGEMENTS}

This study was supported in part by a Grant-in-Aid for Scientific Research from the Japan Society for the Promotion of Science. We thank Hiraku Shida and Akiko Yagi for technical support with immunohistochemistry.
Lu ML, Schneider MC, Zheng Y, Zhang X, Richie JP (2001) Caveolin-1 interacts with androgen receptor. A positive modulator of androgen receptor mediated transactivation. J Biol Chem 276: 13442 - 13451

Lutz MP, Esser IB, Flossmann-Kast BB, Vogelmann R, Luhrs H, Friess H, Buchler MW, Adler G (1998) Overexpression and activation of the tyrosine kinase Src in human pancreatic carcinoma. Biochem Biophys Res Commun 243: $503-508$

Nasu Y, Timme TL, Yang G, Bangma CH, Li L, Ren C, Park SH, DeLeon M, Wang J, Thompson TC (1998) Suppression of caveolin expression induces androgen sensitivity in metastatic androgen-insensitive mouse prostate cancer cells. Nat Med 4: $1062-1064$

Niederhuber JE, Brennan MF, Menck HR (1995) The National Cancer Data Base report on pancreatic cancer. Cancer 76: $1671-1677$

Okamoto T, Schlegel A, Scherer PE, Lisanti MP (1998) Caveolins, a family of scaffolding proteins for organizing 'preassembled signaling complexes' at the plasma membrane. J Biol Chem 273: 5419-5422

Racine C, Belanger M, Hirabayashi H, Boucher M, Chakir J, Couet J (1999) Reduction of caveolin 1 gene expression in lung carcinoma cell lines. Biochem Biophys Res Commun 255: 580-586

Razani B, Zhang XL, Bitzer M, von Gersdorff G, Bottinger EP, Lisanti MP (2001) Caveolin-1 regulates Transforming Growth Factor (TGF)- $\beta /$ SMAD signaling through an interaction with the TGF- $\beta$ type I receptor. $J$ Biol Chem 276: $6727-6738$

Sener SF, Fremgen A, Menck HR, Winchester DP (1999) Pancreatic cancer: a report of treatment and survival trends for 100,313 patients diagnosed from 1985-1995, using the National Cancer Database. J Am Coll Surg 189: $1-7$

Sobin LH, Wittekind Ch (eds) (1997) UICC TNM classification of malignant tumors (5th edn) New York: John Wiley

Suzuki T, Suzuki Y, Hanada K, Hashimoto A, Redpath JL, Stanbridge EJ, Nishijima M, Kitagawa T (1998) Reduction of caveolin-1 expression in tumorigenic human cell hybrids. J Biochem (Tokyo) 124: $383-388$

Timme TL, Goltsov A, Tahir S, Li L, Wang J, Ren C, Johnston RN, Thompson TC (2000) Caveolin-1 is regulated by c-myc and suppresses c-mycinduced apoptosis. Oncogene 19: $3256-3265$

Wiechen K, Diatchenko L, Agoulnik A, Scharff KM, Schober H, Arlt K, Zhumabayeva B, Siebert PD, Dietel M, Schafer R, Sers C (2001a) Caveolin-1 is down-regulated in human ovarian carcinoma and acts as a candidate tumor suppressor gene. Am J Pathol 159: 1635-1643

Wiechen K, Sers C, Agoulnik A, Arlt K, Dietel M, Schlag PM, Schneider U (2001b) Down-regulation of caveolin-1, a candidate tumor suppressor gene, in sarcomas. Am J Pathol 158: 833-839

Yamamoto M, Ohashi O, Saitoh Y (1998) Japan Pancreatic Cancer Registry: current status. Pancreas 16: $238-242$

Yang G, Truong LD, Timme TL, Ren C, Wheeler TM, Park SH, Nasu Y, Bangma CH, Kattan MW, Scardino PT, Thompson TC (1998) Elevated expression of caveolin is associated with prostate and breast cancer. Clin Cancer Res 4: $1873-1880$

Yang G, Truong LD, Wheeler TM, Thompson TC (1999) Caveolin-1 expression in clinically confined human prostate cancer: A novel prognostic marker. Cancer Res 59: 5719-5723 